\title{
Serum C-reactive Protein and Neutrophil/Lymphocyte Ratio After Neoadjuvant Radiotherapy in Soft Tissue Sarcoma
}

\author{
MIO YANAGISAWA ${ }^{1 *}$, ALICIA A. GINGRICH ${ }^{1 *}$, SEAN JUDGE ${ }^{1}$, CHIN-SHANG LI $^{2}$, \\ NANA WANG ${ }^{3}$, STEVEN W. THORPE ${ }^{4}$, AMANDA R. KIRANE ${ }^{1}$, \\ RICHARD J. BOLD ${ }^{1}$, ARTA M. MONJAZEB ${ }^{5}$ and ROBERT J. CANTER ${ }^{1}$ \\ ${ }^{1}$ Division of Surgical Oncology, Department of Surgery, UC Davis School of Medicine, Sacramento, CA, U.S.A.; \\ ${ }^{2}$ Department of Public Health Sciences, Division of Biostatistics, University of California, Davis, CA, U.S.A.; \\ ${ }^{3}$ Department of Statistics, University of California, Davis, CA, U.S.A.; \\ ${ }^{4}$ Department of Orthopedic Surgery, UC Davis School of Medicine, Sacramento, CA, U.S.A.; \\ ${ }^{5}$ Department of Radiation Oncology, UC Davis School of Medicine, Sacramento, CA, U.S.A.
}

\begin{abstract}
Background/Aim: The predictive value of serum $C$-reactive protein $(C R P)$ and neutrophil/lymphocyte $(N / L)$ ratio in soft tissue sarcoma (STS) patients receiving neoadjuvant radiotherapy (RT) has not been analyzed. Patients and Methods: From 2007 to 2015, we identified 98 STS patients from a prospective database. Using multivariate analysis, we analyzed CRP and N/L ratios as predictors of overall survival (OS). Results: Mean age of patients was 59 years, $46 \%$ were female, and $55 \%$ of tumors were located at the extremity. A total of 15 histologies were represented. Fifty percent received preoperative RT. Except for extremity location, characteristics were similar between the preoperative $R T$ and upfront surgery cohorts, including baseline CRP levels and N/L ratios. Multivariate analysis of upfront surgery revealed histological grade, tumor size, and baseline $N / L$ ratio to be predictors of $O S$, while for preoperative $R T$, baseline $C R P$ and $N / L$ ratio were not predictive. Conclusion: Baseline CRP and N/L ratio did not predict poor clinical outcome in STS patients receiving neoadjuvant $R T$.
\end{abstract}

This article is freely accessible online.

Presented in part at the 70th Annual Society for Surgical Oncology Cancer Symposium, Seattle, WA, March 2017.

*These Authors contributed equally to this study.

Correspondence to: Robert J. Canter, MD, UC Davis Cancer Center, 4501 X Street, Suite 3010, Sacramento, CA 95817, U.S.A. Tel: +1 9167345907, Fax: +1 9167035267, e-mail: rjcanter@ucdavis.edu

Key Words: C-reactive protein, CRP, N/L ratio, pre-operative radiotherapy, soft tissue sarcoma, survival.
Recent advances in cancer biology and immunology have focused significant attention on the ability of systemic inflammation to initiate and promote malignancy and alter the host's response to cancer (1). Although the precise mechanisms of how changes in inflammatory pathways alter the tumor microenvironment (TME) and promote tumor growth and metastasis remain complex and incompletely characterized, multiple studies have demonstrated that biomarkers of inflammation, such as serum C-reactive protein (CRP) and neutrophil/lymphocyte (N/L) ratio yield important prognostic information for adverse oncologic outcome in a variety of cancers, including soft tissue sarcoma (STS) (2-6).

$\mathrm{C}$-reactive protein (CRP) is a non-specific serum acutephase inflammatory marker which appears to be primarily secreted by hepatocytes under the influence of interleukin-6 (IL-6) and is driven by tumor-associated tissue inflammation (7). N/L ratio is a calculated index based on levels of circulating neutrophil and lymphocyte counts. N/L ratio is thought to reflect ongoing inflammation thereby skewing the immune repertoire to a pro-tumorigenic neutrophil-driven state and decreased cytotoxic lymphocyte state (8). Elevated serum CRP and N/L ratios (individually and in combination) have previously been identified as independent predictors of worse overall survival (OS) in STS patients (2-5). Importantly, however, all or nearly all these STS patients were treated with upfront surgery with selective adjuvant radiotherapy (RT) and chemotherapy in these studies.

To date, there have been no studies that investigate the predictive potential of CRP and N/L ratio in STS patients receiving neoadjuvant RT. This is an important consideration as RT is thought to alter the inflammatory milieu of the TME. Therefore, we sought to analyze the prognostic value of CRP and N/L ratio in STS patients receiving neoadjuvant $\mathrm{RT}$. 
Table I. Characteristics of entire cohort $(N=98)$.

\begin{tabular}{lc}
\hline Characteristic & Number $(\%)$ \\
\hline Age at Diagnosis (mean \pm SD) & $59.0 \pm 17.9$ \\
Female & $45(46 \%)$ \\
Male & $53(54 \%)$ \\
Caucasian & $72(74 \%)$ \\
Asian & $10(10 \%)$ \\
Hispanic & $13(13 \%)$ \\
Black & $3(3 \%)$ \\
AJCC Stage 2 & $24(24 \%)$ \\
AJCC Stage 3 & $74(76 \%)$ \\
Extremity & $54(55 \%)$ \\
Retroperitoneal & $18(18 \%)$ \\
Trunk/Body Wall & $20(20 \%)$ \\
Other & $6(6 \%)$ \\
Histologic Grade & \\
Intermediate & $20(20 \%)$ \\
High & $78(80 \%)$ \\
HGUPS $\ddagger$ & $35(36 \%)$ \\
Liposarcoma* & $18(18 \%)$ \\
Leiomyosarcoma & $8(8 \%)$ \\
Other** & $37(38 \%)$ \\
Maximal Tumor Size, cm & \\
(median, range) & $9.5(0.7-60.0)$ \\
Margin Status & \\
R0 & $84(86 \%)$ \\
R1 & $10(10 \%)$ \\
R2 & $2(2 \%)$ \\
Baseline CRP, mg/dL (mean $\pm S D)$ & $3.3 \pm 5.6$ \\
Baseline N/L ratio (mean $\pm S D)$ & $4.5 \pm 4.8$ \\
Status at Last Follow-Up & $51(52 \%)$ \\
No evidence of disease & $16(16 \%)$ \\
Alive with disease & $2(2 \%)$ \\
Dead of other causes & $29(30 \%)$ \\
Dead of disease & \\
\hline
\end{tabular}

\#Includes 3 GU, 2 GI, and 1 head and neck. ¥High grade undifferentiated pleomorphic sarcoma. *Includes 11 dedifferentiated liposarcomas, 3 myxoid liposarcomas, 2 myxoid/round cell liposarcomas, and 2 pleomorphic liposarcomas. **Includes 8 synovial sarcomas, 8 myxofibrosarcomas, 5 angiosarcomas, 3 Ewing's/PNET, 3 extraskeletal osteosarcomas, 2 MPNST, 2 solitary fibrous tumors, 2 extraskeletal chondrosarcomas, 1 epithelioid, 1 PEComa, 1 rhabdomyosarcoma, 1

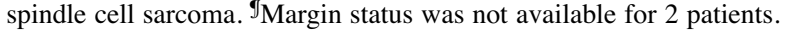

\section{Materials and Methods}

Patients. From November 2007 to December 2015, we identified 108 patients with intermediate or high-grade STS of all anatomic sites who underwent surgical resection. Ten patients who received preoperative chemotherapy were excluded, leaving a total of 98 patients for this study. Following approval by the Institutional Review Board, clinical, pathologic, and treatment data were reviewed and analyzed, including age, gender, tumor location, stage, histologic type, maximal tumor diameter, histologic grade, tumor depth, margin status, and receipt of upfront surgery versus neoadjuvant RT.
Table II. Clinical and pathological characteristics of the preoperative $R T$ and upfront surgery cohorts.

\begin{tabular}{|c|c|c|c|}
\hline Variable & $\begin{array}{c}\mathrm{RT} \\
(\mathrm{N}=49)\end{array}$ & $\begin{array}{l}\text { Upfront surgery } \\
\qquad(\mathrm{N}=49)\end{array}$ & $p$-Value \\
\hline Age $($ mean \pm SD $)$ & $60.2 \pm 17.8$ & $57.7 \pm 18.2$ & 0.49 \\
\hline Female & $24(49 \%)$ & $21(43 \%)$ & 0.69 \\
\hline \multicolumn{4}{|l|}{ Male } \\
\hline Caucasian & $35(71.4 \%)$ & $37(75.5 \%)$ & 0.77 \\
\hline Asian & $5(10.2 \%)$ & $5(10.2 \%)$ & \\
\hline Hispanic & $8(16.3 \%)$ & $5(10.2 \%)$ & \\
\hline Black & $1(2 \%)$ & $2(4.1 \%)$ & \\
\hline AJCC Stage 2 & $7(14.3 \%)$ & $17(34.7 \%)$ & 0.03 \\
\hline AJCC Stage 3 & $42(85.7 \%)$ & $32(65.3 \%)$ & \\
\hline Extremity & $37(75.5 \%)$ & $17(34.7 \%)$ & 0.0002 \\
\hline Retroperitoneal & $6(12.2 \%)$ & $12(24.5 \%)$ & \\
\hline Trunk/Body Wall & $6(12.2 \%)$ & $14(28.6 \%)$ & \\
\hline High grade histology & $38(77.6 \%)$ & $40(81.6 \%)$ & 0.80 \\
\hline HGUPS $\ddagger$ & $20(40.8 \%)$ & $15(30.6 \%)$ & 0.15 \\
\hline Liposarcoma* & $10(20.4 \%)$ & $8(16.3 \%)$ & \\
\hline Leiomyosarcoma & $1(2.0 \%)$ & $7(14.3 \%)$ & \\
\hline Other** & $18(36.7 \%)$ & $19(38.8 \%)$ & \\
\hline $\begin{array}{l}\text { Maximal tumor size } \\
\text { (median, range) }\end{array}$ & $\begin{array}{l}11.5 \\
(3.5-30.0)\end{array}$ & $\begin{array}{l}8.6 \\
(0.7-60.0)\end{array}$ & 0.09 \\
\hline $\begin{array}{l}\text { Baseline CRP, mg/dL } \\
(\text { mean } \pm \mathrm{SD})\end{array}$ & $2.3 \pm 4.3$ & $4.3 \pm 6.5$ & 0.10 \\
\hline $\begin{array}{l}\text { Baseline } \mathrm{N} / \mathrm{L} \text { ratio } \\
(\text { mean } \pm \mathrm{SD})\end{array}$ & $3.9 \pm 5.2$ & $5.0 \pm 4.4$ & 0.16 \\
\hline
\end{tabular}

$\ddagger$ High grade undifferentiated pleomorphic sarcoma. *Includes 11 dedifferentiated liposarcomas, 3 myxoid liposarcomas, 2 myxoid/round cell liposarcomas, and 2 pleomorphic liposarcomas. **Includes 8 synovial sarcomas, 8 myxofibrosarcomas, 5 angiosarcomas, 3 Ewing's/PNET, 3 extraskeletal osteosarcomas, 2 MPNST, 2 solitary fibrous tumors, 2 extraskeletal chondrosarcomas, 1 epithelioid, 1 PEComa, 1 rhabdomyosarcoma, 1 spindle cell sarcoma.

Tumor sites included extremity (at or distal to the shoulder/axilla, and at or distal to the buttock/groin), trunk, retroperitoneal and visceral tumors. Histologic grade (using a three-tiered system) and histologic diagnosis were determined as described previously (9). For purposes of statistical analysis, we limited our analysis to four histology categories, including "other" which represented a composite of 12 subtypes. Distant-recurrence free (DRFS) and overall survival (OS) were calculated as described previously $(10,11)$. OS was calculated from the time of diagnosis to date of death or last known follow-up.

Statistical analyses. Summary statistics were reported as mean \pm standard deviation with median (range) where appropriate. We utilized Cox proportional hazards regression analysis to study the relationship between OS and inflammatory biomarkers at baseline and the pre and post-RT values for the subgroup who received RT. The model was adjusted for covariates associated with OS. Hazard ratios (HR) estimated from the Cox models were reported as relative risks with 95\% confidence intervals (CIs). Survival curves were constructed using the Kaplan-Meier method. Given our sample size, we used median values to categorize patients into "high" and "low" CRP and N/L groups, respectively. Statistical 
Table III. Multivariate analysis of predictors of overall survival.

\begin{tabular}{|c|c|c|c|c|}
\hline \multirow[t]{2}{*}{ Variable } & \multicolumn{2}{|c|}{ Preoperative RT $(\mathrm{N}=49)$} & \multicolumn{2}{|c|}{ Upfront Surgery $(\mathrm{N}=49)$} \\
\hline & Hazard Ratio $(95 \% \mathrm{CI})$ & $p$-Value & Hazard Ratio (95\%CI) & $p$-Value \\
\hline Age & $0.97(0.92-1.01)$ & 0.13 & $1.03(0.997-1.05)$ & 0.08 \\
\hline Tumor Size & $1.07(0.92-1.25)$ & 0.38 & $1.04(0.98-1.09)$ & 0.21 \\
\hline High Grade Histology & $2.01(0.30-24.74)$ & 0.38 & $0.99(0.11-99.9)$ & 0.99 \\
\hline \multicolumn{5}{|l|}{ Histology } \\
\hline HGUPS* & $2.01(0.38-10.68)$ & 0.69 & $1.15(0.31-4.26)$ & 0.69 \\
\hline Leiomyosarcoma & $2.28\left(0.01-10^{7}\right)$ & & $0.62(0.12-3.34)$ & \\
\hline Liposarcoma & $0.47(0.05-4.41)$ & & $0.45(0.10-2.11)$ & \\
\hline Baseline CRP & $1.06(0.97-1.15)$ & 0.19 & $1.16(1.05-1.29)$ & 0.003 \\
\hline Baseline N/L Ratio & $0.80(0.55-1.18)$ & 0.26 & $0.97(0.89-1.05)$ & 0.40 \\
\hline
\end{tabular}

Table IV. Multivariate analysis of predictors of distant-recurrence free survival.

\begin{tabular}{|c|c|c|c|c|}
\hline \multirow[t]{2}{*}{ Variable } & \multicolumn{2}{|c|}{ Preoperative RT $(\mathrm{N}=49)$} & \multicolumn{2}{|c|}{ Upfront Surgery $(\mathrm{N}=49)$} \\
\hline & Hazard Ratio (95\%CI) & $p$-Value & Hazard Ratio (95\%CI) & $p$-Value \\
\hline Age & $0.99(0.96-1.01)$ & 0.27 & $1.01(0.98-1.03)$ & 0.61 \\
\hline Tumor Size & $1.07(0.98-1.17)$ & 0.11 & $1.06(1.00-1.12)$ & 0.05 \\
\hline High Grade Histology & $1.67(0.48-5.83)$ & 0.42 & $5.67(0.68-46.86)$ & 0.11 \\
\hline \multicolumn{5}{|l|}{ Histology } \\
\hline HGUPS* & $0.66(0.19-2.34)$ & 0.71 & $1.84(0.53-6.43)$ & 0.05 \\
\hline Leiomyosarcoma & $1.48\left(0.01-10^{7}\right)$ & & $1.70(0.38-7.49)$ & \\
\hline Liposarcoma & $0.40(0.08-1.88)$ & & $0.06(0.01-0.57)$ & \\
\hline Baseline CRPI & $0.96(0.84-1.10)$ & 0.54 & $1.02(0.96-1.08)$ & 0.49 \\
\hline Baseline N/L Ratio & $1.04(0.98-1.11)$ & 0.18 & $1.08(0.96-1.22)$ & 0.20 \\
\hline
\end{tabular}

*High-grade undifferentiated pleomorphic sarcoma; ${ }^{9} \mathrm{C}$-reactive protein.

analyses were performed using SAS version 9.2 (SAS Institute Inc., Cary, NC). A $p<0.05$ was considered statistically significant.

\section{Results}

The patient and tumor characteristics of our cohort are depicted in Table I. 55\% of tumors were located on the extremity, with $20 \%$ trunk and $18 \%$ retroperitoneal. The median tumor size was $9.5 \mathrm{~cm}$, and undifferentiated pleomorphic sarcoma was the most common histology (36\%), followed by liposarcoma $(18 \%)$ and leiomyosarcoma (8\%). "Other" histologies accounted for $38 \%$. A total of 49 patients received neoadjuvant RT and 49 patients received upfront surgery. Fourteen (29\%) of the upfront surgery patients received adjuvant RT.

Baseline characteristics for the neoadjuvant RT and upfront surgery cohorts are displayed in Table II. With the exception of site and AJCC stage, baseline characteristics were similar. Importantly, neoadjuvant RT and upfront surgery patients demonstrated similar baseline CRP levels and $\mathrm{N} / \mathrm{L}$ ratios.
With a median follow-up of 31.8 months, the 5-year OS was $51.8 \pm 7.8 \%$. Multivariate analysis revealed baseline N/L ratio to be a significant predictor of $\mathrm{OS}$ in patients receiving upfront surgery. However, baseline N/L ratio was not a significant predictor of OS in patients receiving neoadjuvant RT. Additionally, baseline CRP was not associated with OS in either cohort (Table III). When analyzing DRFS, an elevated baseline N/L ratio was associated with a worse outcome on univariate analysis in patients receiving upfront surgery. However, on multivariate analysis, neither N/L ratio nor CRP was predictive of worse DRFS for either cohort (Table IV).

Changes in $\mathrm{N} / \mathrm{L}$ ratio and $\mathrm{CRP}$ before and after neoadjuvant RT are depicted in Figure 1. We observed that preoperative RT was associated with an increase in both CRP $(+1.00 \pm 3.00, p<0.0001)$ and $\mathrm{N} / \mathrm{L}$ ratio $(+1.64 \pm 1.07$, $p<0.0001)$. As shown in Figure 2, Kaplan-Meier analysis demonstrated a trend $(p=0.06)$ for worse OS among upfront surgery patients with high CRP, but there was no difference in OS between high and low CRP among patients receiving 

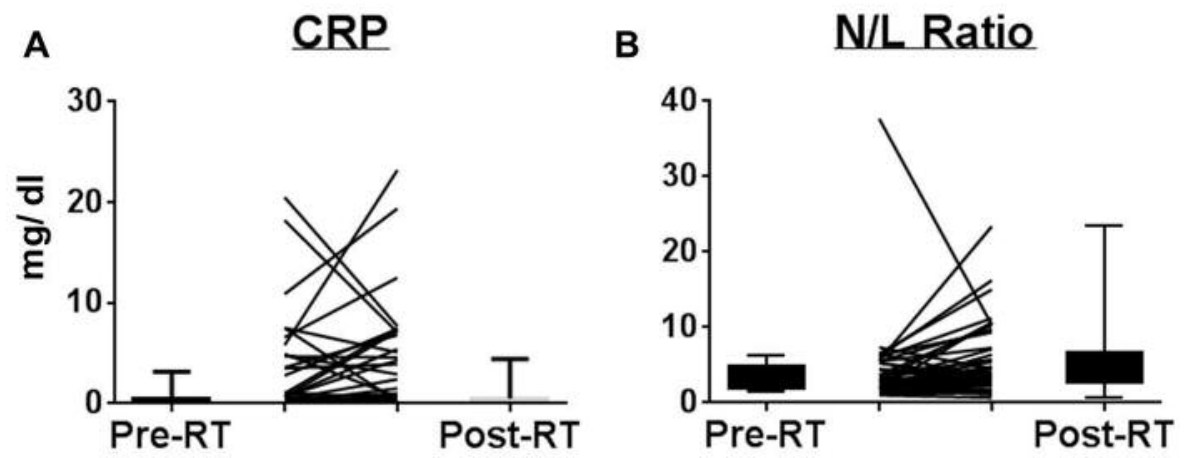

Figure 1. Serum CRP and N/L ratio levels before and after preoperative radiotherapy.
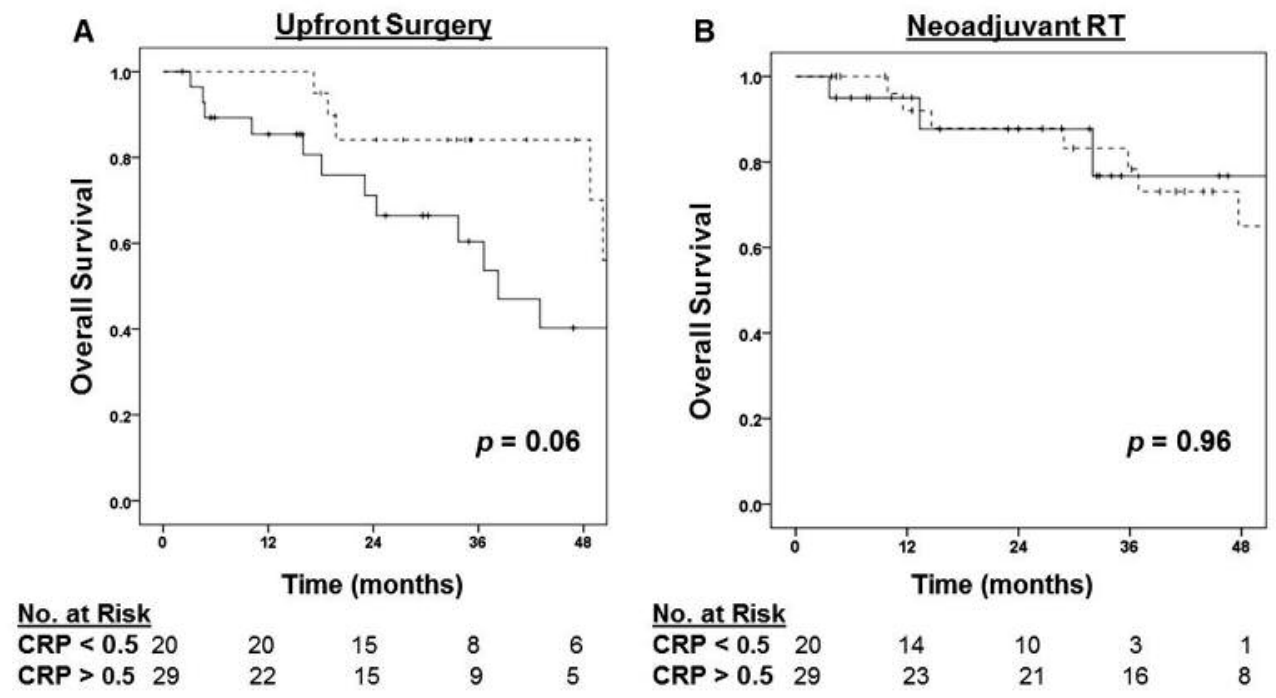

\section{-... Baseline CRP $<0.5$ \\ - Baseline CRP > 0.5}

Figure 2. Overall survival among upfront surgery and preoperative radiotherapy cohorts stratified by C-reactive protein levels. Patients were stratified based on the median value of the entire cohort as the cut-point.

neoadjuvant RT. Similarly, OS was worse among upfront surgery patients with an elevated N/L ratio (Figure 3 ), but there was no difference in OS among patients receiving preoperative RT stratified by baseline N/L ratio.

\section{Discussion}

Similar to other solid tumors, previous work has clearly demonstrated the prognostic value of CRP and N/L ratio in patients with STS (6). Nakamura et al., for example, observed that an elevated CRP $>0.3 \mathrm{mg} / \mathrm{dl}$ before initial treatment was an independent predictor of worse event-free survival by approximately $30 \%$ in 102 patients with localized STS (2). In a subsequent report, Nakamura et al., analyzed 142 STS patients and found that elevated CRP and an N/L ratio $>2.3$ independently predicted shorter disease-specific survival (87\% 5-year OS for normal CRP and low N/L ratio compared to $46 \%$ for elevated CRP and high NLR) (3). More recently, Nakamura explored CRP levels in patients with metastatic STS and also found that CRP was an independent prognostic factor for shorter disease-specific survival (12). Similarly, Szkandera et al. evaluated 304 STS patients, 


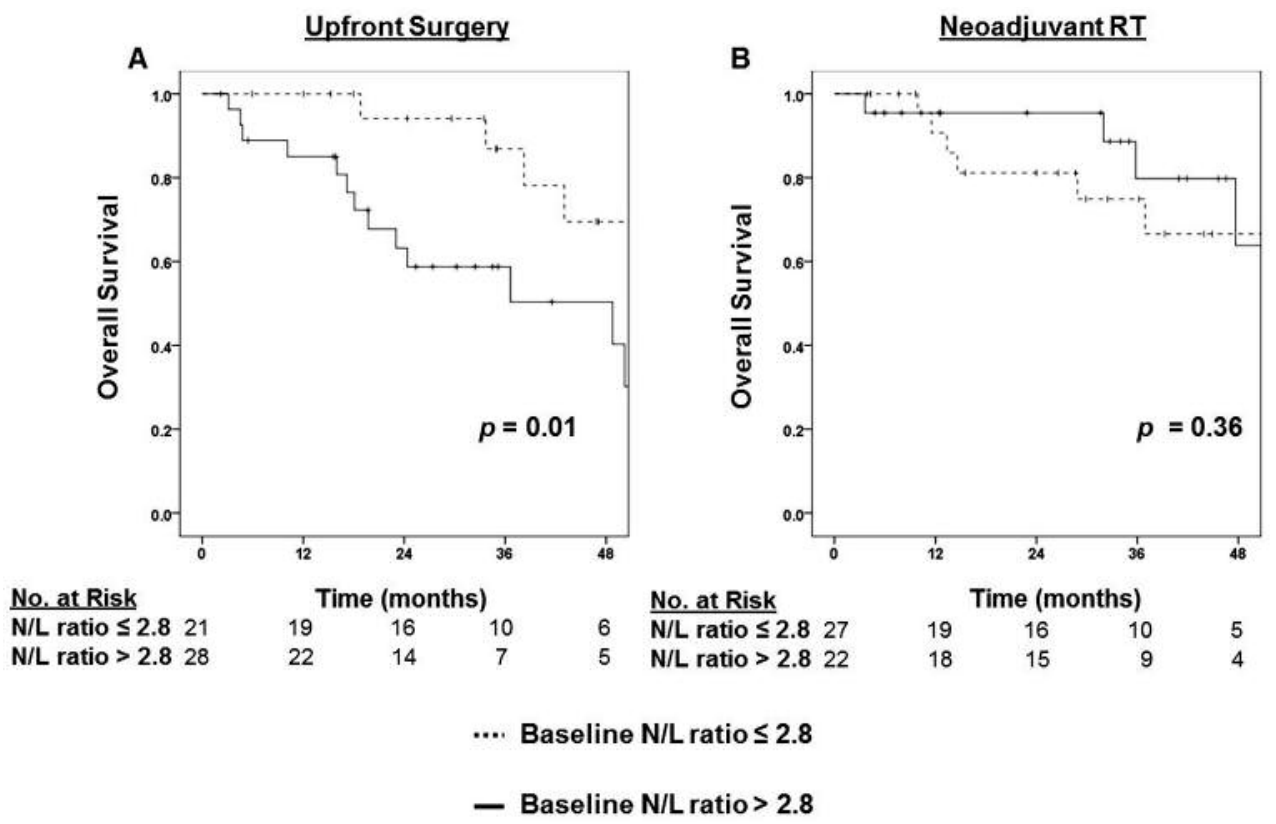

Figure 3. Overall survival among upfront surgery and preoperative radiotherapy cohorts stratified by N/L ratios. Patients were stratified into high and low neutrophil/lymphocyte ratios based on the median value for the entire cohort as the cut-point.

identifying elevated preoperative CRP $\geq 0.69 \mathrm{mg} / \mathrm{dl}$ as an independent predictor of worse survival (5). In a separate report from the same group, Szkandera analyzed 260 STS patients and found that an N/L ratio $\geq$ approximately 3.5 was associated with worse time to STS recurrence and OS (4).

Yet, despite these consistent data regarding the adverse oncological impact of elevated CRP and N/L ratio, the overwhelming majority of studies (in STS and other solid tumors) have evaluated these markers in the setting of either upfront surgery or metastatic disease. In both Nakamura reports, no patients received neoadjuvant $\mathrm{RT}$, and the majority $(\sim 80 \%)$ was treated with surgery as monotherapy. In the Szkandera studies, all patients were treated with upfront surgery, but a larger fraction $(\sim 60 \%)$ received adjuvant RT. To our knowledge, our study is, therefore, the first to evaluate the impact of CRP and N/L levels on STS patients receiving neoadjuvant $\mathrm{RT}$, comparing them to patients receiving upfront surgery (and adjuvant RT in approximately $30 \%$ of cases). Our results uphold the association between elevated N/L ratio with decreased OS in patients undergoing upfront surgery. However, in patients receiving neoadjuvant $\mathrm{RT}$, we observed a novel finding of no association between elevated CRP and elevated N/L ratio for both OS and DRFS. Therefore, our findings fill a gap in the literature, which although potentially unexpected, are nevertheless novel and clinically relevant.

Although our data do not address the underlying mechanism for why neoadjuvant RT may decouple the association of elevated CRP and N/L ratio with worse STS outcome, one broad hypothesis is that neoadjuvant RT may impact the inflammatory milieu of the TME in a different manner than upfront surgery and thereby alter the interaction of these bio-markers with outcome (12-15). For example, pre-clinical studies suggest that complex inflammatory and cell-signaling cascades secondary to RT may mediate a strong immune response which can counteract and in some cases overcome the local immunosuppression of the TME (16). These observations and further studies to understand the impact of RT on the TME are especially pertinent with the ongoing development of novel radiotherapy regimens such as hypofractionated RT and innovative energy sources such as protons and carbon ions (17). Although the question of how local RT-induced changes translate to systemic changes in inflammatory markers and possible antiinflammatory mediators remains unanswered, our data support a potential impact of neoadjuvant RT on these inflammatory pathways.

Other studies have suggested that metabolic pathways are significant in sarcoma oncogenesis and progression, and the impact of anti-metabolites and RT on the TME are worthy of further investigation. For example, Igarashi et. al observed decreased survival of osteosarcoma cells and xenografts after administration of a methionine inhibitor (18). As RT is known to produce reactive oxygen species and dysregulate key machinery of cellular metabolism, it is reasonable to hypothesize an interaction causing alterations in the 
inflammatory environment. Interestingly, the same group studied the therapeutic effects of caffeine and valproic acid and found similar anti-metabolic and anti-tumor effects (19). Other groups such as Ahn et al. have explored the antitumorigenic effects of compounds such as sphingosine, a metabolite of sphingolipids, which has known antiinflammatory properties (20). They showed that administration of sphingosine to alveolar rhabdomyosarcoma cell lines caused a dose dependent inhibition of growth and acceleration of cell death. Therefore, the interaction of CRP and $\mathrm{N} / \mathrm{L}$ ratio in the setting of other anti-inflammatory mediators requires further study, and comparative proteomics approaches using novel mass spectrometry techniques may reveal insights into these complex protein interactions (21).

Despite the potential novelty of our results, it is important to acknowledge our study's limitations. Firstly, it is a retrospective, single-institution study which has a smaller sample size relative to other studies evaluating CRP and N/L ratios in STS. As is typical of STS studies, our analysis included a heterogeneous cohort of numerous histologic subtypes and tumor locations, and this created an imbalance in baseline characteristics between our neoadjuvant RT and upfront surgery cohorts which may have biased our results. However, despite these differences in clinical/pathological characteristics, the baseline CRP and N/L ratios were statistically similar between the two cohorts. Since these markers were the principal predictor variables for our analysis, the similarity in baseline values between the groups suggests that the associations we observed with the outcomes of interest were not unduly confounded by selection bias between the RT and upfront surgery groups. However, we acknowledge that our study may have been underpowered to detect modest but potentially significant differences in baseline CRP and N/L ratio levels between the cohorts. With a larger sample size, especially among the neoadjuvant RT patients, it is possible that CRP levels and N/L ratios may show potentially significant associations with oncological outcomes as studies of STS patients receiving upfront surgery have shown. Further research of this question appears warranted.

Ultimately, we found that baseline and post-treatment N/L ratio and CRP do not correlate with survival in STS patients receiving neoadjuvant $\mathrm{RT}$. Thus, the utility of $\mathrm{N} / \mathrm{L}$ ratio and CRP as predictors of poor clinical outcome may not apply in these patients. In fact, patients with elevated CRP and N/L ratio at diagnosis may be good candidates for neoadjuvant $\mathrm{RT}$, although these decisions should remain individualized.

\section{Conflicts of Interest}

The Authors have no financial relationships to disclose. The project described was supported by the National Center for Advancing Translational Sciences (NCATS), National Institutes of Health (NIH), through grant \#UL1 TR001860.

\section{References}

1 Hanahan D and Weinberg RA: Hallmarks of cancer: the next generation. Cell 144(5): 646-674, 2011.

2 Nakamura T, Matsumine A, Matsubara T, Asanuma K, Uchida A and Sudo A: Clinical significance of pretreatment serum Creactive protein level in soft tissue sarcoma. Cancer 118(4): 1055-1061, 2012.

3 Nakamura T, Matsumine A, Matsubara T, Asanuma K, Uchida A and Sudo A: The combined use of the neutrophil-lymphocyte ratio and C-reactive protein level as prognostic predictors in adult patients with soft tissue sarcoma. J Surg Oncol 108(7): 481-485, 2013.

4 Szkandera J, Absenger G, Liegl-Atzwanger B, Pichler M, Stotz M, Samonigg H, Glehr M, Zacherl M, Stojakovic T and Gerger A: Elevated preoperative neutrophil/lymphocyte ratio is associated with poor prognosis in soft-tissue sarcoma patients. Br J Cancer 108(8): 1677-1683, 2013.

5 Szkandera J, Gerger A, Liegl-Atzwanger B, Absenger G, Stotz M, Samonigg H, Maurer-Ertl W, Stojakovic T, Ploner F and Leithner A: Validation of the prognostic relevance of plasma Creactive protein levels in soft-tissue sarcoma patients. $\mathrm{Br} \mathrm{J}$ Cancer 109(9): 2316-2322, 2013.

6 Templeton AJ, McNamara MG, Seruga B, Vera-Badillo FE, Aneja P, Ocana A, Leibowitz-Amit R, Sonpavde G, Knox JJ and Tran B: Prognostic role of neutrophil-to-lymphocyte ratio in solid tumors: a systematic review and meta-analysis. J Natl Cancer Inst 106(6): dju124, 2014.

7 Gabay C and Kushner I: Acute-phase proteins and other systemic responses to inflammation. N Engl J Med 340(6): 448454, 1999.

8 Zhang $\mathrm{X}$, Zhang W, Yuan X, Fu M, Qian H and Xu W: Neutrophils in cancer development and progression: Roles, mechanisms, and implications (Review). Int J Oncol 49(3): 857867, 2016.

9 Canter RJ, Borys D, Olusanya A, Li CS, Lee LY, Boutin RD, Christensen SD, Tamurian RM and Monjazeb AM: Phase I trial of neoadjuvant conformal radiotherapy plus sorafenib for patients with locally advanced soft tissue sarcoma of the extremity. Ann Surg Oncol 21(5): 1616-1623, 2014.

10 Canter RJ, Martinez SR, Tamurian RM, Wilton M, Li CS, Ryu J, Mak W, Monsky WL and Borys D: Radiographic and histologic response to neoadjuvant radiotherapy in patients with soft tissue sarcoma. Ann Surg Oncol 17(10): 2578-2584, 2010.

11 Shah D, Borys D, Martinez SR, Li CS, Tamurian RM, Bold RJ, Monjazeb A and Canter RJ: Complete pathologic response to neoadjuvant radiotherapy is predictive of oncological outcome in patients with soft tissue sarcoma. Anticancer Res 32(9): 39113915, 2012.

12 Nakamura T, Katagiri H, Shido Y, Hamada S, Yamada K, Nagano A, Yamada S, Tsukushi S, Ishimura S, Matsumine A, Sudo A and Nishida Y: Analysis of factors for predicting survival in soft-tissue sarcoma with metastatic disease at initial presentation. Anticancer Res 37(6): 3137-3141, 2017.

13 Galon J, Angell HK, Bedognetti D and Marincola FM: The continuum of cancer immunosurveillance: prognostic, predictive, and mechanistic signatures. Immunity 39(1): 11-26, 2013.

14 Formenti SC and Demaria S: Radiation therapy to convert the tumor into an in situ vaccine. Int $\mathrm{J}$ Radiat Oncol Biol Phys 84(4): 879-880, 2012. 
15 Demaria S and Formenti SC: Radiation as an immunological adjuvant: current evidence on dose and fractionation. Front Oncol 2: 153, 2012.

16 Demaria S and Formenti SC: Role of T lymphocytes in tumor response to radiotherapy. Front Oncol 2: 95, 2012.

17 Imai R, Kamada T, Araki N, and The Working Group for Bone and Soft-Tissue Sarcomas: Clinical efficacy of carbon ion radiotherapy for unresectable chondrosarcomas. Anticancer Res 37(12): 6959-6964, 2017.

18 Igarashi K, Kawaguchi K, Kiyuna T, Miyake K, Murakami T, Yamamoto N, Hayashi K, Kimura H, Miwa S, Tsuchiya H and Hoffman RM: Effective metabolic targeting of human osteosarcoma cells in vitro and in orthotopic nude-mouse models with recombinant methioninase. Anticancer Res 37(9): 40814084, 2017.

19 Igarashi K, Kawaguchi K, Kiyuna T, Murakami T, Yamamoto N, Hayashi K, Kimura H, Miwa S, Tsuchiya H and Hoffman RM: Antimetastatic efficacy of the combination of caffeine and valproic acid on an orthotopic human osteosarcoma cell line model in nude mice. Anticancer Res 37(8): 1005-1011, 2017.
20 Ahn EH, Lee MB, Seo DJ, Lee J, Kim Y and Gupta K: Sphingosine induces apoptosis and down-regulation of MYCN in PAX3-FOXO1-positive alveolar rhabdomyosarcoma cells irrespective of TP53 mutation. Anticancer Res 38(1): 71-76, 2018.

21 Dimas K, Tsimplouli C, Anagnostopoulos AK, Mahaira L, Iliopoulou E, Perez S, Vougas K and Tsangaris GT: The proteome profile of two cell lines and their xenografts isolated from a patient with clear cell sarcoma (soft tissue melanoma). Cancer Genomics Proteomics 5(3-4): 175-237, 2008.
Received January 1, 2018

Revised January 31, 2018

Accepted February 1, 2018 